\title{
Affordances and Constraints of Virtual Healthcare: Students' Experiences Accessing Online Physician
}

\author{
Jackie Nelson $^{1}$, Viola Manokore ${ }^{1^{*}}$, Chris Carvalho ${ }^{2}$, Cindy Boucher ${ }^{1}$ and Sheldon Kallio ${ }^{3}$ \\ ${ }^{1}$ NorQuest College, Edmonton, AB T5J 1L6, Canada \\ ${ }^{2}$ Carveira Group Consulting, Edmonton, AB T5E 6N2, Canada \\ ${ }^{3}$ Three Hive Consulting Inc., Canada
}

\section{Abstract}

Background: Students at a community college in Alberta were invited to participate in this project that offered access to virtual healthcare. Prior to inception of this project, the government medical insurance did not cover virtual healthcare for its members. However, at the end of this project, virtual healthcare was considered for coverage due to challenges created by COVID-19 pandemic. The main purpose of this study was to explore the affordances and constraints of a virtual healthcare platform as reported by the students who used the online primary healthcare services.

Methods: Students enrolled in the fall 2019 and winter 2020 term at the community college were invited to enrol in the project and given access to the virtual healthcare services provided by a Canadian company. Over 15,000 emails were send to the students at the college. Students who consented and volunteered to participate were enrolled to access the online physicians through the virtual healthcare company's platform. 430 participants registered to be enrolled for the project. A total of 142 consultations were made. Participants completed a survey on their experiences soon after their consultations.

Results: The findings from the survey show that the students found the platform to be convenient, especially given the circumstances created by the COVID-19 pandemic. The students' average satisfaction with the virtual healthcare services was 4.8 out of 5 stars. Students found the platform to be user friendly, convenient to students in rural areas and newcomers to Canada, and better than visiting clinics. However, students indicated that they will only access online physicians outside the project if insurance providers cover the services.

Conclusions: Findings suggest that virtual healthcare is convenient to patients especially given the healthcare access challenges that were a result of COVID-19 pandemic. The virtual healthcare will also reduce inequalities in health access of people who reside in rural and remote areas where clinics are at a distance.

\section{Introduction}

There are many healthcare lessons that were learned as a result of COVID-19 pandemic. One lesson pertains to the limitation of healthcare access for non-COVID-19 illnesses as nations focused on the pandemic. Given the infectious nature of the disease, patients with non-urgent medical requirements and non-COVID-19 symptoms had reduced access to physical healthcare facilities as providers enforced physical distancing measures. Pre-planned non-urgent surgeries were delayed and providers experienced reduced capacity due to limitations on physical gatherings (e.g., meetings, waiting rooms) and enhanced environmental preparation procedures (e.g., cleaning, PPE). That approach created a gap in providing healthcare to non-COVID-19 individuals. For countries that have comprehensive legislation on virtual healthcare, that gap could be filled by use of remote consultations using information technologies. However, Canada has not fully utilized the opportunities of virtual healthcare. While virtual providers were permitted to operate, legislation was not amended to facilitate health insurances to pay service providers for virtual healthcare. At the initial stage of the project-despite the need for virtual healthcare, Alberta Health Care Insurance Plan (AHCIP) was among those major insurers that allowed virtual providers to operate within the regions, but did not cover costs for virtual healthcare. As a result, only those individuals who could afford pay-for-service fee to use virtual healthcare platforms ultimately had an option of virtual healthcare in Alberta. However, because of COVID-19, regulations were relaxed to cover virtual healthcare for AHCIP members. This study explores students' experiences using a virtual platform to access non-emergency healthcare provided by online physicians through a Canadian company, Maple. In this paper, the patients were the students who utilized the virtual healthcare platform.

Information technology provides opportunities for improving access to healthcare and reduces pressure on in-person services. Telemedicine is about delivering different forms healthcare to those who need it, without their having to physically visit the physician's office. Services provided include health education, diagnosis, treatment and administrative activities [1]. In a survey done by Ipsos [2] in 27 countries, only $10 \%$ of the participants indicated that they had used telemedicine. This suggests that virtual healthcare is a huge opportunity area globally. It has a potential of improving accessibility and minimizing inequalities of quickly accessing quality healthcare, especially in communities that have limited access.

Virtual healthcare is crucial in times of pandemics and epidemics of infectious diseases. Remote primary healthcare is a logical approach as it helps to avoid spread of infectious diseases as physician scan make a diagnosis remotely and advise patients on next steps. "Corresponding Author: Dr Viola Manokore, NorQuest College, NorQuest -Health and Community Studies, Edmonton, AB T5J 1L6, Canada; E-mail: viola. manokore@norquest.ca

Citation: Nelson J, Manokore V, Carvalho C, Boucher C, Kallio S (2020) Affordances and Constraints of Virtual Healthcare: Students' Experiences Accessing Online Physician. Int J Nurs Clin Pract 7: 327. doi: https://doi.org/10.15344/2394$4978 / 2020 / 327$

Copyright: (C) 2020 Manokore. This is an open-access article distributed under the terms of the Creative Commons Attribution License, which permits unrestricted use, distribution, and reproduction in any medium, provided the original author and source are credited. 
In addition, it helps to avoid infected patients from going to their physician's office where they could meet vulnerable populations and spread the disease. Asymptomatic patients-a particular concern demonstrated with COVID-19 could also spread the infection to the physician. As a result, the physician might unknowingly infect their patients and families. The potential for exponential infectious disease spread could be easily mitigated by providing virtual healthcare, contributing to the health and safety of both healthcare systems and communities.

Beyond the mitigation of infectious disease spread, virtual healthcare addresses other pressing issues regarding healthcare access and capacities. Maniatopoulos et al. [3] pointed out that with the increase in aging population, there is need to increase easy access to healthcare and that virtual healthcare can be convenient for aging citizens. The authors argued that virtual deliveries would provide easy access to healthcare. The European Commission for Information Society and Media (ECISM) developed a plan in which virtual healthcare has demonstrated benefits for distant diagnosis, monitoring and management. ECISM notes virtual health contributes to a shift from symptom-based care to preventative and person-centred care, with benefits for ageing citizens, early diagnosis, empowerment among citizens [4].

In addition to benefits to individuals, virtual healthcare holds significant potential with regard to healthcare systems management and efficiencies. Vimalananda et al. [5] conducted a systematic review to explore the scale-up viability of eConsult in some provinces in Canada. eConsult is a type of asynchronous healthcare consultation that aims to alleviate patient wait times, which is one of Canada's greatest challenge in healthcare. The authors found that e-consults could lead to improved management of patients in a primary care settings (no need for specialist visits), reduced time to completion for patient workups, improvements to wait times and provider perceptions of wait times, and shorter referral times [5].

Liddy et al. [6] highlighted key factors that influence the scaling up of eConsult identified by the Canadian National eConsult Policy Think Tank. The factors include remuneration of healthcare providers, issues related to the privacy of patient information and medical professions licensing between jurisdictions [7]. The Canadian Medical Association (CMA) (n.d) [8] noted that technologies to deliver healthcare from a distance have not been fully adopted by healthcare systems in developed countries. While it acknowledged that virtual healthcare would help improve access to communities in rural and remote areas CMA pointed to numerous barriers to effective implementation within existing healthcare systems. The barriers highlighted include issues related to licensing of the virtual healthcare providers, payment for services, quality of care provided, and training, among other issues. CMA noted that some industrialized countries have frameworks for virtual healthcare and hence the need for Canada to do the same. In February 2020, CMA set up a taskforce, including four working groups, to explore and develop a framework about how virtual healthcare could be implemented in Canada. A survey in 2018 on behalf of CMA mirrors the global Ipsos survey, showing that less than $10 \%$ of the Canadian population has used virtual consultations and $69 \%$ would want to use virtual healthcare if it were available (CMA, n.d.).

There is an information gap with regard to utilization, affordances and constraints associated with virtual healthcare in Canada and Alberta in particular. In the systematic review cited above, four provinces that had identified eConsult scaling up were Ontario, Quebec, Manitoba, and Newfoundland and Labrador [5]. The prioritization of remote consult has been slow in the current study context of Alberta and the current study provides some evidence to validate the importance moving forward with virtual healthcare systems. Within an environment of provincial budget constraints, at-capacity healthcare services, large underserved rural regions, this project is important as it provides baseline data on healthcare access, quality of virtual care, and insights on how virtual healthcare could provide alternatives to physical walk-in clinics and physician's visits. The data collected could also inform policy makers. Government health services require this data to determine if virtual healthcare should be permanently funded in the same way as face-to-face consultations.

The current study, "virtual health project," was implemented at a community college in Alberta. The project was designed to provide the students with access to non-emergency primary healthcare services offered by Maple through their virtual platform. The virtual health care project paid for students' virtual consultations through funding that was provided by Alberta Innovates, an arms-length research and development government agency. Students travel to post-secondary from around the world, and are often removed from the support of their primary care physician. Students often live without personal transportation, experience time constraints, and may be unfamiliar with local health access procedures. Access to primary healthcare for students can therefore be challenging, especially during an infectious disease pandemic. The project was aimed to make virtual healthcare access available to all students regardless of whether they had a family physician, and was designed to address access issues prior to the emergence of COVID-19. After the onset of COVID-19, students were also impacted by access restrictions related to pandemic control, further restricting their ability to seek primary care. The virtual project provided students with non-COVID-19 illness to have access to primary virtual healthcare. The project was also designed to gather insights on the feasibility and viability of using virtual healthcare services in Alberta. This project gathered evidence on the relevance, access, and practicality of using virtual healthcare services. The virtual healthcare project was guided by the following broad objectives:

1. To provide timely access and healthcare services to the community college students who consented to participate in the project.

2. To validate use of virtual healthcare application in Alberta.

3. To explore acceptance of virtual healthcare by the students at the community college.

\section{Methods}

\section{Study context}

The community college where this project was implemented has a diverse population of students. More than half of the student population were born outside Canada. The college offers foundational courses to new immigrants; academic upgrading courses for high schools, certificate and diploma programs. Some students do not have a family physician they can easily get in-touch with if they needed primary healthcare, and others do not have their own primary healthcare physician at all. The project provided opportunities for students to access healthcare services at any time by online application or any audio device. 
Citation: Nelson J, Manokore V, Carvalho C, Boucher C, Kallio S (2020) Affordances and Constraints of Virtual Healthcare: Students' Experiences Accessing Online Physician. Int J Nurs Clin Pract 7: 327. doi: https://doi.org/10.15344/2394-4978/2020/327

\section{Project participants}

Students who had at least grade eight English reading levels were invited to participate in the study. Email addresses from student administrative records (both personal and institutional addresses) were used to recruit students enrolled at the college in fall 2019 and winter 2020 semesters. In October 2019 and February 2020, fifteen thousand email invitations were sent to about seven thousand students with qualifying enrolment records. Students who completed the enrolment form and consented to have their contact details shared with the virtual healthcare services provider (Maple) were enrolled into the online virtual application.

A total of 511 students completed the enrolment form. Of these, 430 students consented to have their contact details shared with the virtual healthcare service provider. The healthcare provider sent invitations to enrolled students to register for the services. Consultations were available throughout the day and night, including weekends. Consultations between students and the online physicians were private, and services provided between enrolment and the completion of the virtual consultation are beyond the scope of the study. Additionally, for reasons related to privacy, the research team did not collect information from medical records or specific details on who accessed the services after registration. During consultations, participants used communication technologies of their choice that were included as part of the virtual care platform (video, text or telephone consultation). At the time of writing, a total of 142 consults had been completed. After consultation, students received an automated invitation to participate in a survey to share their experiences. Fifty-one students completed the survey and their responses are reported in this paper

\section{Description of the platform}

The community college where participants were recruited partnered with Maple, a Canadian based company that provides 24/7 access to a healthcare professionals through a virtual platform. As indicated, the Maple (n.d.) platform is accessible from a mobile phone, tablet, or computer. During consultations, a patient is able to communicate directly with an online physician for primary healthcare and receive a diagnosis, prescription, laboratory requisitions or referral. The website is secure and when a patient logs in to the virtual platform, they are matched to a physician licensed in Alberta. The physician then reviews the patient's request and interacts with the patient using audio, text or video. The virtual platform securely stores patients' medical records, which are then made accessible to the patient, as well as to healthcare providers during future virtual visits with Maple virtual physicians.

\section{Data collection tools}

Students' invitations included a link to participant information letter and consent form to enable students to understand the data collection and participation procedures, provide their enrolment information, and consent to participant in the study. Following consultation, 52 students completed a point of service survey to provide feedback about their experiences with the services provided. Survey completion was voluntary.

\section{Results and Discussion}

Descriptive statistics on the nature of the consultations was drawn from monthly reports supplied to the research team by the service provider. The usage reports included: average waiting time, average consultation time, number of referrals, times of consultation, and ratings. Thematic analyses of open-ended questions were drawn from the follow-up point of service survey. Themes were identified that represent experiences of students as they used the virtual healthcare application. Table 1 below shows some indicators that are based on the 142 consultations made by the students.

\section{Constraints of virtual healthcare}

Figure 1 summaries the affordances and constraints of using virtual healthcare as reported by the participants. Although the feedback was positive overall, four out of 51 responses indicated some constraints of using the virtual healthcare platform. One participant said "it would be nice if there was a list of services that they are able to perform. It was a waste of my time and consultations to find out they can't help." The participant possibly had a consult that required a referral for a follow-up physical examination or specialist consultations not

\begin{tabular}{|c|c|}
\hline Key Indicator & Comment \\
\hline \multicolumn{2}{|l|}{ Utilization } \\
\hline Total Consults (\#) 142 & \multirow{4}{*}{$\begin{array}{l}\text { Majority of the consultations were done during 'normal' walk-in clinic hours. About } 12.5 \% \text { of the } \\
\text { consultations were done overnight. It is evident that the virtual healthcare provided participants } \\
\text { with opportunities to easily access healthcare overnight and ease the burden of visiting emergency } \\
\text { rooms, which are the only available services in these hours. The high frequency of use during } \\
\text { hours where face-to-face services are available suggests that virtual care can reduce in-person } \\
\text { traffic and capacity issues at busy locations by distributing these services to available physicians, } \\
\text { regardless of their location. Data also indicates a demand for alternative ways to access physicians } \\
\text { during normal business hours. }\end{array}$} \\
\hline Weekday (7am - Midnight EST) 91 & \\
\hline Weekend / Holiday (7am - Midnight EST) 33 & \\
\hline Overnight (Midnight - 7am EST) 18 & \\
\hline \multicolumn{2}{|l|}{ Consultation Data } \\
\hline Average Wait Time- 3 minutes & \multirow{5}{*}{$\begin{array}{l}\text { Patients typically drive or take public transit to walk-in clinics and the wait times in Alberta can } \\
\text { be more than one hour. In this project, participants' average wait time was less than two minutes. } \\
\text { Not only would virtual services save time and money for patients, it has a potential of reducing } \\
\text { the load in walk-in clinics and emergency rooms. }\end{array}$} \\
\hline Average Consult Length- 16.7 minutes & \\
\hline Prescriptions Ordered (\#) 107 & \\
\hline Medical Notes Written (\#) 9 & \\
\hline Lab Requisitions (\#) 21 & \\
\hline \multicolumn{2}{|l|}{ Ratings / Feedback } \\
\hline Average Satisfaction (out of 5 stars) 4.8 stars & It was evident from the ratings that participants were satisfied with the virtual healthcare services. \\
\hline
\end{tabular}


Citation: Nelson J, Manokore V, Carvalho C, Boucher C, Kallio S (2020) Affordances and Constraints of Virtual Healthcare: Students' Experiences Accessing Online Physician. Int J Nurs Clin Pract 7: 327. doi: https://doi.org/10.15344/2394-4978/2020/327

available through Maple. The online platform does provide a list of services available that describe the limitations based on the remote format. However, this comment suggests some potential patients are not yet familiar with the capacities of virtual healthcare and providers may need additional education and information strategies to clarify these elements. Another response indicated that "this was great technology; however, it was difficult to get a picture of my throat... [I needed help to] take the picture as I couldn't get a proper picture myself." In this case, the online physician needed a picture so that they could have a closer visual of the patient's symptoms in order to provide an appropriate diagnosis. Patients sometimes experience physical limitations or capacity for technology use, such as using a camera or uploading a photo that might limit their ability to support their diagnosis through technology. This limitation is more likely to impact vulnerable populations, such as seniors, people with disabilities, or those with limited access to and experience with technology. The third constraint highlighted by one participant is related to issues of internet connectivity. The participant said, "I like the idea of it. Communication back and forth was a bit slow. Could have been the connection." The response highlights the need for reliable Internet connections to support good patient experiences-both on the patient and the physician end. Particularly in remote regions, Internet connectivity will pose an ongoing challenge for virtual health access. The fourth constraint highlighted in one response shows that the patient was not able to clearly describe their condition. The student stated, "it seemed limiting in the way where I couldn't really communicate properly when it came to detailing my problem." This is a potential problem that might lead to patient frustration or a potential misdiagnosis when a patient inadvertently misrepresents their illness. However, the same issues may arise during an in-person visit when a patient cannot explain their condition.

\section{Affordances of virtual healthcare}

Overall, participants in this study had positive experiences with the virtual healthcare program. Table 2 below summarises students' experiences after they used the virtual healthcare application. Participants' responses also indicate how they compared "callin" versus "walk-in" clinics in cases where they do not make prior appointments. In this project, patients/participants did not need to make a prior appointment to consult a virtual physician. Rather, they just "called-in," waited for a couple of minutes for an available physician, and entered their consultation. The participants also indicated that they would use virtual physician outside the project if the virtual healthcare was available with no cost to the patient and covered by health insurance.

Virtual healthcare improves access to care in remote and rural communities. It helps to reduce inequalities in healthcare associated with physical distances between facilities and patients. Participants in this project also indicated that virtual healthcare works well for those who do not drive and do not have a family physician. However, as pointed out by CMA (n.d.), there is need for connectivity of patient data and the healthcare providers that include physician, pharmacist, hospitals, long-term care facilities and specialists so as to provide holistic care that includes a continuity of care between virtual and physical services. Quality healthcare is important to provide good patient outcomes and high satisfaction rates among participants indicates that virtual healthcare does provide good quality care and can meaningfully contribute to an effective health system.

While two thirds of the participants that used Maple indicated that they had a family physician, $28 \%$ of respondents did not have a family

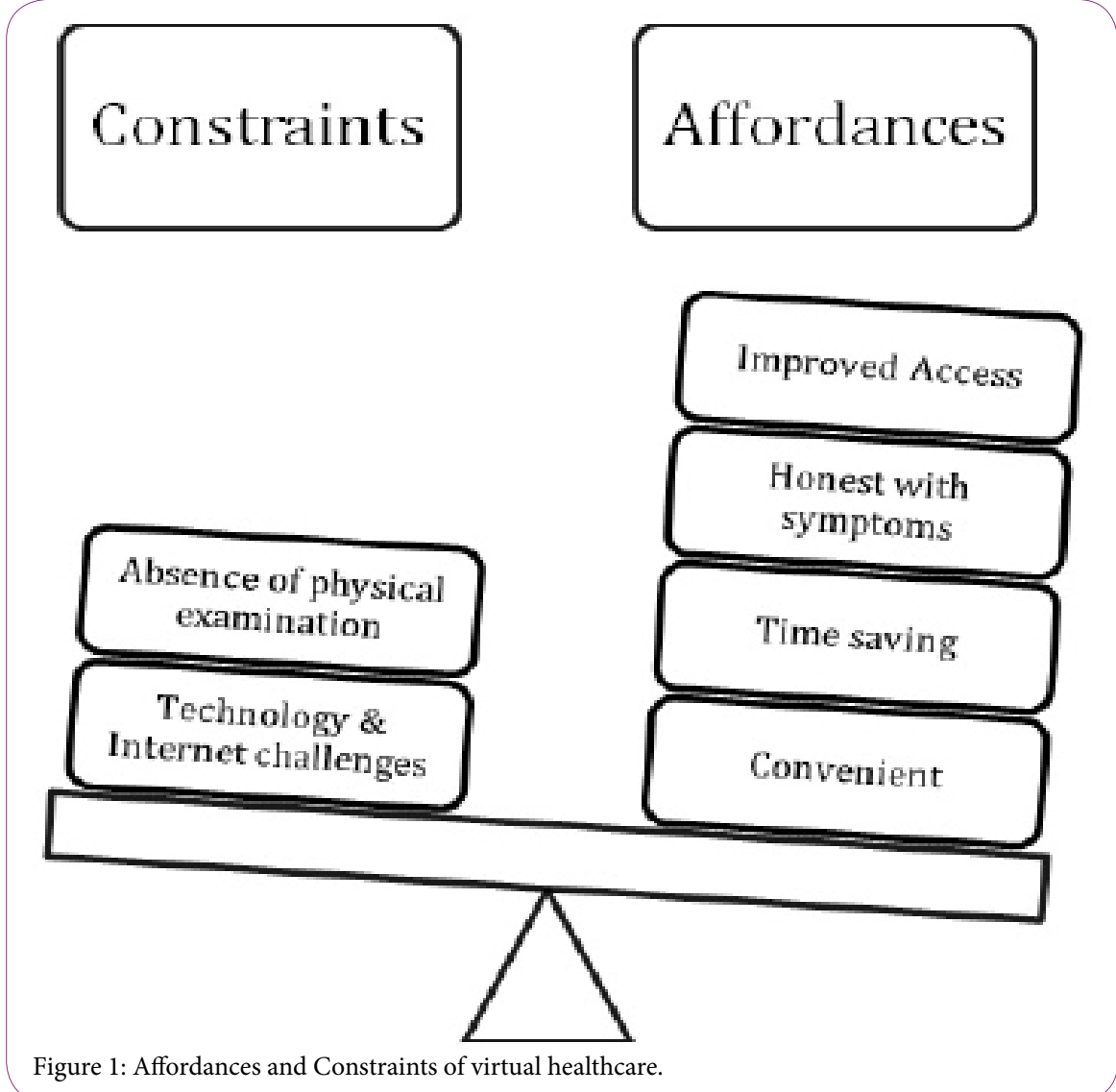




\begin{tabular}{|c|c|c|}
\hline Main Theme & Quotations from students & Comment \\
\hline Convenience & $\begin{array}{l}\text { "I absolutely love this, it is easy and convenient for me, looking forward to } \\
\text { use it in future" } \\
\text { "This was so unbelievably convenient. I needed a simple prescription } \\
\text { renewal and am too busy to go to my family doctor. I would love to continue } \\
\text { to use this service!" } \\
\text { "The experience was good. It was great that I was able to seek medical help } \\
\text { from a medical professional without having to leave my house rather than } \\
\text { just using the internet to google my symptoms so I find that awesome" } \\
\text { "I had a minor health complaint and it was something I probably would } \\
\text { have waited to go see the Physician for as it was difficult for me to book } \\
\text { appointments with my busy schedule. It was nice to get a quick answer/ } \\
\text { diagnosis in a few minutes of my down time! }\end{array}$ & $\begin{array}{l}\text { It is evident from participants' feedback that } \\
\text { they found their consultations with the virtual } \\
\text { physician convenient. Participants in this project } \\
\text { were students who are busy with their studies } \\
\text { and other obligations. They indicated that having } \\
\text { easy and quick access was suitable as it took only } \\
\text { a few minutes to get what they needed without } \\
\text { accessing in-person facilities. }\end{array}$ \\
\hline Save time & $\begin{array}{l}\text { "Very easy to use and if you don't have time to sit in a physicians office for } 30 \\
\text { minutes to an hour it's a great way to save time and money" } \\
\text { "This experience was awesome! Way better than waiting in a waiting room } \\
\text { for hours on end" } \\
\text { "I really enjoyed being able to access this service. It was nice, quick and easy. } \\
\text { I was able to get the two prescriptions faxed directly to my pharmacy. It was } \\
\text { nice to have access to it when I otherwise would not be able to get into my } \\
\text { family physician or have time to sit in a medicentre. } \\
\text { I would be interested to see how it would work regarding anti depressants or } \\
\text { any controlled substances and getting a prescription for that." } \\
\text { "The technology is helpful especially to people who don't drive, or if } \\
\text { someone is not physically stable enough to get out of the house to see a } \\
\text { physician. Also, this is better when all you need is a minor prescription. You } \\
\text { don't have to book an appointment days ahead and wait for } 30 \text { mins for a } \\
\text { 5-minute appointment" }\end{array}$ & $\begin{array}{l}\text { Getting healthcare services through walk-in } \\
\text { clinics can be time consuming. They would take } \\
\text { time to walk, cycle, taxi, drive or take public } \\
\text { transport to their nearest walk-in clinic. When } \\
\text { they get there, patients would wait for a about an } \\
\text { hour before they could consult with the physician. } \\
\text { During times of infectious diseases pandemics/ } \\
\text { epidemics waiting in physical clinics could expose } \\
\text { patients to infections and contribute toward } \\
\text { exponential spread of the disease. Technology } \\
\text { helped with quick access and saved time for the } \\
\text { participants. }\end{array}$ \\
\hline $\begin{array}{l}\text { Open to share } \\
\text { symptoms }\end{array}$ & $\begin{array}{l}\text { "The technology was very convenient, I felt like I could provide honest and } \\
\text { unbiased answers o the computer screen... The prescriptions were quite } \\
\text { handy and it was super easy to get them sent to me, no ambiguity through } \\
\text { messy hand-writing or anything of the sorts. The biggest limitation was } \\
\text { evidence, such as taking pictures of your throat. Maybe have a phone app } \\
\text { that can upload direct while you're in a conversation on your computer so } \\
\text { you wouldn't have to change what device you're chatting on. Or have pre- } \\
\text { emptive requirements such as if you have a sore throat take a picture before } \\
\text { hand." } \\
\text { "My experience was great! I was able to speak with a physician quickly and } \\
\text { with it being digital, I found that I actually felt more comfortable talking } \\
\text { to the physician as opposed to being face to face. All necessary questions } \\
\text { were asked and answered honestly, the physician was friendly and took his } \\
\text { time, when my medication was prescribed he provided details. I think if this } \\
\text { virtual service was available to everyone, it would be super beneficial!" }\end{array}$ & $\begin{array}{l}\text { Not all patients feel comfortable enough to share } \\
\text { their symptoms with healthcare providers. In } \\
\text { this project, participants were using technology } \\
\text { to communicate with the virtual physician. } \\
\text { Feedback from participants suggests that virtual } \\
\text { healthcare removed barriers of communication as } \\
\text { patients provided honest answers. Honest answers } \\
\text { from patients also assistwith proper diagnosis and } \\
\text { treatment. }\end{array}$ \\
\hline $\begin{array}{l}\text { Improved } \\
\text { access to } \\
\text { immigrants\& } \\
\text { rural patients }\end{array}$ & $\begin{array}{l}\text { "Really very helpful like me new comers to Canada who don't know how to } \\
\text { get medical help. Really wonderful service." } \\
\text { "Great for rural residents.... Specially [for a] working person who has hard } \\
\text { to find time." }\end{array}$ & $\begin{array}{l}\text { Virtual healthcare is an essential service for } \\
\text { patients who reside far away from walk-in clinics. } \\
\text { They get to consult with a virtual physician at any } \\
\text { time }\end{array}$ \\
\hline $\begin{array}{l}\text { Professionalism } \\
\text { of care } \\
\text { providers }\end{array}$ & $\begin{array}{l}\text { "Very professional and nice/helpful." } \\
\text { "Nice conversation with your Physician." } \\
\text { "It was efficient and quick, the physician was very thorough and the use of } \\
\text { the video chat made it feel like a regular trip to the physician." }\end{array}$ & $\begin{array}{l}\text { Despite physical distance, the participants were } \\
\text { satisfied with the quality of care they received. }\end{array}$ \\
\hline
\end{tabular}


Citation: Nelson J, Manokore V, Carvalho C, Boucher C, Kallio S (2020) Affordances and Constraints of Virtual Healthcare: Students' Experiences Accessing Online Physician. Int J Nurs Clin Pract 7: 327. doi: https://doi.org/10.15344/2394-4978/2020/327

physician. It is also important to note that the project was active both before COVID-19 and during the pandemic, when non-COVID-19 patients had additional to their ability to access to healthcare. In Alberta, about $18 \%$ of the population does not have a family physician [4]. While we are unable to determine whether the community college's population as a whole is less likely to have a family physician, the data demonstrates that those who used the Maple platform were nearly two times as likely to not have a family physician compared to Alberta's population, suggesting the platform provided access to those who did not have a family physician. This analysis also suggests, however, that for two thirds of participants, using Maple represents a divergence from ongoing continuity of care with a family physician.

\section{Other virtual healthcare considerations}

\section{Licensingand remuneration}

Liddy et al. [6] argued that there is need for a Canadian framework that clarifies how services will be offered and also the registration of virtual healthcare providers. In our studies, students indicated that they would use the services if they are paid for by health insurance. Virtual services have historically seen limited coverage by government insurers in Canada, and although accommodations were implemented during the global pandemic, those services are at risk of under-funding or removal post-pandemic. Coverage for virtual health consultations also varies among private health insurers, as coverage by those policies is not mandated.

\section{Education and training}

Education about best use of virtual healthcare if also important to prepare users. Patients and providers need to understand which healthcare access point best meets a patient's current need so that they can maximize the available opportunities and be prepared for that interaction. Patient comments suggesting they wish they knew pictures were required, or which services issues could be addressed in the virtual format, illustrate the patient side of this dynamic. . Training is also needed in schools of medicine, nursing, pharmacy, allied health, and other disciplines to teach carers how to effectively provide virtual healthcare. This includes not only health delivery training, but also technology training to help ensure that virtual healthcare providers are be equipped to assist patients who experience physical or technological barriers.

\section{Conclusions and Recommendations}

The main recommendation is for medical insurance agencies to pay for their members who opt to use virtual healthcare. Virtual healthcare services have a potential of alleviating some of the following issues and improve patient outcomes:

1. Waiting times- patients in this study did not have to make prior appointments. The wait time average was only three minutes. Under current in-person services, patients who visit walk-in clinics would wait for about an hour to see a physician.

2. Access inequalities- patients who reside in remote areas have to travel long distances to access primary healthcare. Virtual healthcare has a potential to provide quick and reliable access to patients who need it.

3. Reduce infections - COVID-19 pandemic impacted patients with non-Covid-19 conditions' access to healthcare. Virtual healthcare provides a safe approach in primary healthcare to individuals, without exposing others to infectious diseases. Infectious diseases are always present in healthcare systems, and reduced exposure to ailments like flu and cold could reduce all disease transmission, therefore reducing healthcare system burdens

4. Comfortable in sharing online- patients who might be hesitant to share their symptoms during in-person visits would have options to use technology to mediate that interaction. For some patients, this may support honest communication and help in proper diagnosis.

5. Ease of use- Although technology-mediated healthcare is not easier for all patients, for many people this approach offers a solution to barriers that make it difficult to access physicians. Learning and using the technology is relatively straight-forward for many potential users.

\section{Competing Interests}

The author declare that there is no competing interests regarding the publication of this article.

\section{Funding}

The project was supported by Alberta Innovates grant number 20180308 in support of technology driven practice and training in healthcare.

\section{References}

1. Wootton $R$, Bonnardot $L$ (2015) Telemedicine in low-resource settings. Front Public Health 3: 3

2. Ipsos (2018) Global views on healthcare.

3. Maniatopoulos G, McLoughlin I, Wilson R, Martin M (2009) Developing Virtual Healthcare Systems in Complex MultiAgency Service Settings: the OLDES Project. Electronic Journal of e-Government 7: 163 -170.

4. Clarke J (2016) Difficulty accessing health care services in Canada. Statistics Canada (ECISM). European Commission for Information Society and Media. Information Society and Health: Linking European Policies, Brussels: European Commission.

5. Vimalananda VG, Gupte G, Seraj SM, Orlander J, Berlowitz D, et al. (2015) Electronic consultations (e-consults) to improve access to specialty care: a systematic review and narrative synthesis. J Telemed Telecare 21: 323-330.

6. Liddy C, Moroz I, Joschko J, Horsley T, Kuziemsky C, et al. (2018) Using an Integrated Knowledge Translation (IKT) Approach to Enable Policy Change for Electronic Consultations in Canada. Healthc Policy 14: 19-29.

7. Liddy $C$, McKellips F, Armstrong CD, Afkham A, Fraser-Roberts $L$, et al. (2017) Improving access to specialists in remote communities: a crosssectional study and cost analysis of the use of eConsult in Nunavut. Int J Circumpolar Health 76: 1323493

8. Canadian Medical Association. Virtual Care in Canada: Discussion Paper. CMA Health Summit.

This article was originally published in a special issue:

Community-Based Participatory Research Practices-Vol II

Handled by Editor(s):

Prof. Reiko Okahisa

Department of Community Health Nursing

Tokushima University

Japan 